\title{
SUPERCONDUCTING CONTACTS TO A TWO-DIMENSIONAL ELECTRON GAS IN GaAs/AlGaAs-HETEROSTRUCTURES
}

\author{
K.-M.H. Lenssen, M. Matters, C.J.P.M. Harmans, J.E. Mooij \\ Delft University of Technology, Department of Applied Physics, \\ P.O. Box 5046, 2600 GA Delft, The Netherlands \\ M.R. Leys, W. van der Vleuten, J.H. Wolter \\ Eindhoven University of Technology, Department of Applied Physics, \\ P.O. Box 513, 5600 MB Eindhoven, The Netherlands
}

\begin{abstract}
Recently we succeeded in making superconducting contacts of $\mu \mathrm{m}$-scale to the two-dimensional electron gas in GaAs/AlGaAs-heterostructures, in combination with gate structures. The contacts produced by this method are highly transmissive down to very low temperatures. We present the first preliminary results of the resistance measurements at $T=80 \mathrm{mK}$. These give clear evidence for the occurrence of Andreev reflection. Moreover a strong influence of the gate voltage on the $d V / d I-V$ curves has been observed, which proves the essential role of the semiconductor.
\end{abstract}

\section{INTRODUCTION}

Since the idea of a Josephson field-effect transistor or superconducting transistor was described by T.D. Clark et al. [1], the interest for this device has been growing. It is based on the proximity coupling of two superconducting electrodes [2], connected by a semiconductor. The supercurrent which can flow through the device can be modulated by varying the electron density with a gate voltage.

The devices realized until now $[3,4,5,6,7,8]$ are all operating in the dirty limit, where the electron transport is diffusive. Very different and new effects are expected in the clean limit, where the electron transport is phase coherent. Only recently the availability of very high mobility two-dimensional semiconductors and the state of microfabrication techniques make it possible to achieve this limit. This has resulted in a renewed interest in this subject, both theoretically and experimentally.

In this paper we will first discuss some aspects of the theory for this new class of superconducting devices. In the third section the fabrication of the samples is discussed, followed by the results of the first measurements in section IV.

\section{THEORY}

A superconducting weak link consists of a conducting material (metal or semiconductor), denoted by $\mathrm{N}$, between two superconducting regions $\mathrm{S}$. If there is a good electrical contact at the SN-interfaces, Cooper-pairs can "diffuse"

\footnotetext{
Manuscript received August 24, 1992
}

into the $\mathrm{N}$-region, which results in a finite order parameter. This means that a supercurrent can flow through a semiconductor, if the distance $L$ between the superconducting regions is small enough, i.e. of the order of the normal coherence length

$$
\xi_{\mathrm{N}}=\sqrt{\frac{\hbar D}{2 \pi k_{\mathrm{B}} T}}
$$

where $D$ is the electron diffusion constant and $k_{\mathrm{B}}$ the Boltzmann constant. This formula is valid in the dirty limit $l_{\mathrm{e}}<L\left(l_{\mathrm{e}}\right.$ is the elastic mean free path).

Recently it has been predicted that the critical supercurrent is quantized in the ballistic regime $[9,10]$. It would be interesting to test this with a quantum point contact between two ballistically connected superconducting electrodes.

An other process which can occur in SNS-systems is Andreev-reflection $[11,12]$. An electron in the N-region with an excitation energy $E$ smaller than the superconducting gap $\Delta$ cannot enter the superconductor, because no excitation states are available. The electron will be reflected as a hole with excitation energy $-E$ in exactly the opposite direction with respect to the incoming electron, while a Cooper-pair is created in the superconductor. Since the incoming electron (hole) and the reflected hole (electron) contribute both to a current in the same direction, Andreev-reflection gives rise to an excess current and reduces the interface resistance [13]. In SNS-junctions multiple Andreev-reflection can take place, if $L$ is sufficiently small. This will result in changes in the differential resistance at voltages corresponding to submultiples of the gap ( $2 \Delta / n$ with $n$ integer).

The probability of Andreev-reflection is determined strongly by the transmission of the NS-interface: In particular at interfaces with semiconductors the Schottky-barrier can introduce normal reflection and reduce this probability significantly.

Especially in the clean limit Andreev-reflection is a very powerful concept for describing multiprobe SNSsystems, because it is easily incorporated in a generalized Landauer-Büttiker formalism $[14,15]$. New effects are expected in this ballistic regime, because of the phase coherence of the electrons. For example, because an electron which was reflected at one NS-interface can undergo the 


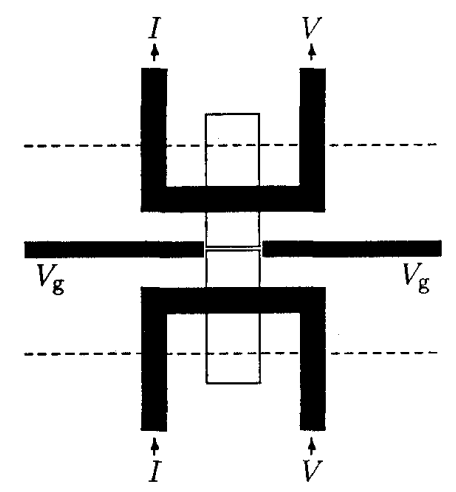

Figure 1: Layout of the centre of the sample; the dashed lines are the boundaries of the etched mesa, the black areas are gold layers, the two rectangles are the $\mathrm{Sn} / \mathrm{Ti}$-contacts

reversed reflection process at the other NS-interface, a periodic motion can arise. This will result in quantization of the energy levels of the excitations and will lead to a Josephson current $[16,17]$.

\section{SAMPLE FABRICATION}

To study the transport in the clean, ballistic regime the condition $l_{\mathrm{e}}>L$ should be fulfilled. Moreover $L$ should be large enough to put split-gate structures between the superconducting contacts. That means $l_{\mathrm{e}}>\xi_{\mathrm{N}} \gtrsim 10^{-6} \mathrm{~m}$. Because of the large mobility $\mu_{\mathrm{e}}$ of the two-dimensional electron gas (2DEG), combined with the excellent gating properties GaAs/AlGaAs-heterostructures are preferred. Moreover the Fermi-wavelength is rather large, which facilitates the study of quantum effects $\left(\lambda_{F} \approx 50 \mathrm{~nm}\right)$. The material we used for the first tests had the following properties: $\mu_{\mathrm{e}}=42 \mathrm{~m}^{2} / \mathrm{Vs}_{\mathrm{s}}$ and electron density $n_{\mathrm{e}}=3.1 \cdot 10^{15} \mathrm{~m}^{-2}$, resulting in $l_{\mathrm{e}}=4 \mu \mathrm{m}$ and $\xi_{\mathrm{N}}=2.7 \mu \mathrm{m}$ at $T=80 \mathrm{mK}$. The material was grown by Molecular Bearn Epitaxy and consists of a GaAs-substrate with a $4 \mu \mathrm{m}$ undoped GaAs-layer, a $20 \mathrm{~mm}$ undoped $\mathrm{Al}_{0.33} \mathrm{Ga}_{0.67}$ As-spacer, a $40 \mathrm{~nm}$ n-doped $\mathrm{Al}_{0.33} \mathrm{Ga}_{0.6} \mathrm{As}$ layer and an $18 \mathrm{~nm}$ undoped GaAs-caplayer.

Fabricating a clean, superconducting, olmic contact to the 2DEG, situated $80 \mathrm{~nm}$ below the surface, is a major problem. Dry etching, to bring the metallic contact closer to the 2DEG by removing the toplayer, will damage the material over considerable distances $(\gtrsim 100 \mathrm{~nm})$ near the etched surfaces. That is why we have chosen a diffusion process. Details of the fabrication will be published elsewhere, so here we only give a short outline.

The layout of the sample is shown in figure 1. Because of the required resolution we used electron beam lithography. To align all the processing steps (one etching and up to three metallization steps) we had to use especially prepared gold markers. The alignment was done by automatic

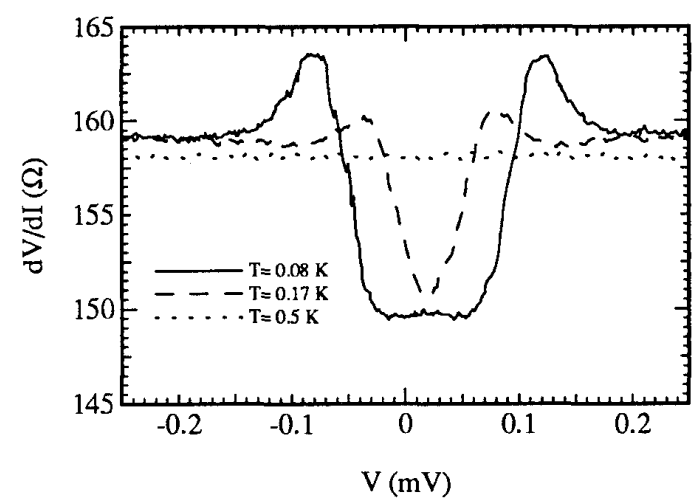

Figure 2: The differential resistance as function of the voltage at different temperatures $\left(V_{\mathrm{g}}=0 \mathrm{~V}\right)$

marker search. The achieved relative alignment turned out to be better than $\approx 30 \mathrm{~nm}$.

The most important process steps are preparing gold markers, mesa-etching for device isolation, $\mathrm{Sn} / \mathrm{Ti}$ metallization and diffusion for the actual contacts and Au-metallization. In this first sample there are gates at the sides of the S-contacts to confine the area where the (super-)current can flow. The distance between the contacts was defined lithographically as $L=1000 \mathrm{~nm}$, the contact width is $10 \mu \mathrm{m}$. Four separated larger contacts were added, to enable tests (e.g. quantum Hall-measurement to test two-dimensionality). The measurements were performed in a dilution refrigerator at $T \approx 80 \mathrm{mK}$. The resistances were measured by a current-biased ac lock-in technique as well by de methods.

\section{RESULTS}

The results of the differential resistance measurements at different temperatures are presented in figure 2 . Because of bonding problems the measurements on this sample were done in a three-terminal configuration. The estimated series resistance is $100-150 \Omega$. A low resistance region exists around zero-voltage, flanked by two peaks. It has been checked by measuring the two contacts separately using the larger contacts, that this is not only a feature of a single contact. At first glance these measurements could be explained by the flow of a supercurrent in the system, taking into account a constant series resistance. However the series resistance turned out to be influenced by applying a gate voltage. Nevertheless the shape is typical for Andreev-reflection in NS-structures. The voltage where the jump in resistance occurs should correspond with $2 \Delta$. In the Blonder-Tinkham-Klapwijkmodel [13] the resistance below the jump should be half the resistance above it (in the clean case). This yields a series resistance of $139 \Omega$. The corresponding gap is $9 \mu \mathrm{eV}$ at $T=80 \mathrm{mK}$, which is possible for Ti. Moreover the typical shape of the curve has disappeared completely at $0.5 \mathrm{~K}$. 
This is in agreement with the critical temperature of $\mathrm{Ti}$ $\left(T_{\mathrm{c}} \approx 0.4 \mathrm{~K}\right)$. No evidence for multiple Andreev-reflection was found; perhaps the mobility of the 2DEG was not high enough to measure this.

At large negative gate-voltages $V_{\mathrm{g}}$ the resistance dip disappears completely. This remarkable effect is not very well understood yet, but it proves that the semiconductor plays an essential role in the origin of the peak structure.

\section{CONCLUSIONS}

The process of $\mathrm{Sn} / \mathrm{Ti}$-diffusion provides lowresistance (highly transmissive) contacts to the 2DEG in GaAs/AlGaAs-heterostructures. At temperatures down to $80 \mathrm{mK}$ the resistance stays very low. The temperature and gate-voltage dependence of the $d V / d I-V$-curves show that Andreev-reflection must take place in the sample. The measurements suggest that $\mathrm{Ti}$ is the relevant superconductor and not $\mathrm{Sn}$. The fact that the bottom of the low resistance region is very flat and does not show a peak near $V=0 \mathrm{~V}$ means that the transmission of the NS-interface is very close to 1 . Therefore these superconducting contacts seem to be very suitable to study SNS-junctions in the clean limit.

\section{ACKNOWLEDGMENT}

We would like to thank the Delft Institute of Microelectronics and Submicron Technology for the use of their facilities. This research was financially supported by the Dutch Foundation for Fundamental Research on Matter (Stichting F.O.M.).

\section{REFERENCES}

[1] T.D. Clark, R.J. Prance and A.D.C. Grassie, "Feasibility of hybrid Josephson field effect transistor", J. Appl. Phys, vol. 51, pp. 2736-2743, May 1980

[2] P.G. de Gennes, "Boundary effects in Superconductors", Rev. Mod. Phys, vol. 36, pp. 225-237, January 1964

[3] H. Takayanagi and T. Kawakami, "Superconducting proximity effect in the native inversion layer of $\operatorname{InAs",}$ Phys. Rev. Lett., vol. 54, pp. 2449-2452, June 1985

[4] T. Nishino, M. Miyake, Y. Harada and U. Kawabe, "Three-terminal superconducting device using a $\mathrm{Si}$ single-crystal film", IEEE Electron Device Lett., vol. EDL-6, pp. 297-299, June 1985

[5] Z. Ivanov, T. Claeson and T. Andersson, "Three terminal Josephson junction with a semiconductor accumulation layer", in Proc. 18th Int. Conf. on Low Temperature Physics, Kyoto, Japan, vol. 3, pp. 1617 1618, August 1987
[6] A.W. Kleinsasser, T.N. Jackson, D. McInturff, F. Rammo, G.D. Pettit and J.M. Woodall, "Superconducting InGaAs junction field-effect transistors with Nb electrodes", Appl. Phys. Lett, vol. 55, pp. 19091911, October 1989

[7] W.M. van Fuffelen, M.J. de Boer and T.M. Klapwijk, "Ultra-thin silicon membranes to study supercurrent transport in crystalline semiconductors", Appl. Phys. Lett., vol. 58, pp. 2438-2440, May 1991

[8] A. Kastalsky, L.H. Greene, J.B. Barner and R. Bhat, "Proximity-effect superconductive tunneling in $\mathrm{Nb}$ on InGaAs/InP/InGaAs heterostructures", Phys. Rev. Lett., vol. 64, pp. 958-961, February 1990

[9] A. Furusaki, H. Takayanagi and M. Tsukada, "Theory of quantum conduction of supercurrent through a constriction", Phys. Rev. Lett., vol. 67, pp. 132-135, July 1991

[10] C.W.J. Beenakker and H. van Houten, "The superconducting quantum point contact", in Proc. Int. Symp. on Nanostructures and Mesoscopic Systems, Santa Fe, U.S.A., May 1991

[11] A.F. Andreev, "The thermal conductivity of the intermediate state in superconductors", Zh. Eksp. Teor. Fiz., vol. 46, pp. 1823-1828, May 1964 [Sov. Phys. JETP, vol. 19, pp. 1228-1231, November 1964]

[12] A.F. Andreev, "Electron spectrum of the intermediate state of superconductors", Zh. Eksp. Teor. Fiz., vol. 49, pp. 655-660, August 1965 [Sov. Phys. JETP, vol. 22, pp. 455-458, February 1966]

[13] G.E. Blonder, M.Tinkham and T.M. Klapwijk, "Transition from metallic to tunneling regimes in superconducting microconstrictions: excess current, charge imbalance, and supercurrent conversion", Phys. Rev. B, vol. 25, pp. 4515-4532, April 1982

[14] R. Kümmel, U. Gunsenheimer and R. Nicolsky, "Andreev scattering of quasiparticle wave packets and current voltage characteristics of superconducting metallic weak links",.Phys. Rev. B, vol. 42, pp. 3992-4009, September 1990

[15] B.J. van Wees, K.-M.H. Lenssen and C.J.P.M. Harmans, "Transmission formalism for supercurrent flow in multiprobe superconductor-semiconductorsuperconductor devices", Phys. Rev. B, vol. 44, pp. 470-473, July 1991

[16] I.O. Kulik, "Macroscopic quantization and the proximity effect in S-N-S junctions", Zh. Eksp. Teor. Fiz, vol. 57, pp. 1745-1759, November 1969 [Sov. Phys. JETP, vol. 30, pp. 944-950, May 1970]

[17] J. Bardeen and. J.L. Johnson, "Josephson current flow in pure superconducting-normal-superconducting junctions", Phys. Rev. B, vol. 5, pp. 72-78, January 1972 